\title{
Neuer Wirkstoff Abaloparatid verhindert Knochenbrüche
}

\begin{abstract}
Das Hormon-Analogon Abaloparatid, das wie Teriparatid den Knochenaufbau fördert, hat in einer randomisierten Studie im US-amerikanischen Ärzteblatt (1) (JAMA 2016; 316: 722-733) die Knochenmineraldichte von postmenopausalen Osteoporose-Patientinnen gesteigert und im Vergleich zu Placebo die Zahl der vertebralen Frakturen deutlich gesenkt. Ein Unterschied zu Teriparatid war eine geringere Rate von Hyperkalzämien. Der Hersteller kann auf eine rasche Zulassung in den USA und in Europa hoffen. Ob er eine kostengünstigere Alternative zu dem hochpreisigen Teriparatid-Präparat anbieten wird, bleibt abzuwarten.
\end{abstract}

Teriparatid, eine auf 34 Aminosäuren verkürzte rekombinante Variante des humanen Parathormons, wurde bereits 2003 in Deutschland zugelassen. Obwohl das Mittel im Gegensatz zu den Bisphosphonaten, die die Knochenresorption hemmen, eine anabole Wirkung hat, sprich den Wiederaufbau von Knochensubstanz fördert, wird es derzeit kaum eingesetzt.

Der Grund liegt in den bis zu 35-fach höheren Therapietageskosten, die in keinem oder wenn überhaupt nur geringen wirtschaftlichen Verhältnis zum zusätzlichen Nutzen stehen. Ein Nutzen ist aber möglicherweise dann gegeben, wenn es aufgrund der Schwere der Erkrankung gelingt, die Fähigkeit zur Selbstversorgung von älteren Menschen zu erhalten und damit eine Pflegebedürftigkeit zu vermeiden. Der gemeinsame Bundesausschuss (G-BA) hat deshalb die Anwendung auf Patienten mit schwerer Osteoporose (mindestens zwei neue Wirbelkörperfrakturen in den letzten 18 Monaten) beschränkt, die Bisphosphonate und Raloxifen nicht vertragen.

Teriparatid, das die Firma Lilly als Forste ${ }^{\circledR}$ anbietet, wird demnächst Konkurrenz von Abaloparatid erhalten, das die Firma Radius aus Waltham, Massachusetts entwickelt hat. Abaloparatid ist ein synthetisches Peptid. Es besteht ebenfalls aus 34 Aminosäuren. Es ahmt aber nicht die Wirkung des Parathormons (PTH), sondern die des PTH-related Protein (PTHrP) nach. Beide Wirkstoffe wirken zwar am gleichen Rezeptor (PTH-Rezeptor Typ 1), allerdings in anderer Weise. Der Unterschied soll darin bestehen, dass Teriparatid neben der anabolen auch eine leichte katabole Wirkung auf den Knochenstoffwechsel hat, was zu einem Anstieg des Kalziumspiegels führt. Diese Nebenwirkung soll bei Abaloparatid nicht vorhanden sein oder wenigstens deutlich schwächer ausfallen.
Die Firma Radius als Hersteller ließ Abaloparatid in einer Phase 3-Studie testen, an der 2463 postmenopausale Frauen an 28 Zentren in zehn Ländern (keine deutsche Beteiligung) teilnahmen. Die Patienten hatten eine verminderte Knochendichte und radiologische Nachweise von mindestens zwei leichten Frakturen (oder wenigstens eine mittelschwere bis schwere Fraktur).

Sie wurden im gleichen Verhältnis auf drei Studienarme randomisiert, in denen sie tägliche subkutane Injektionen von Teriparatid, Ababolparatid oder Placebo erhielten. Ein Pla-

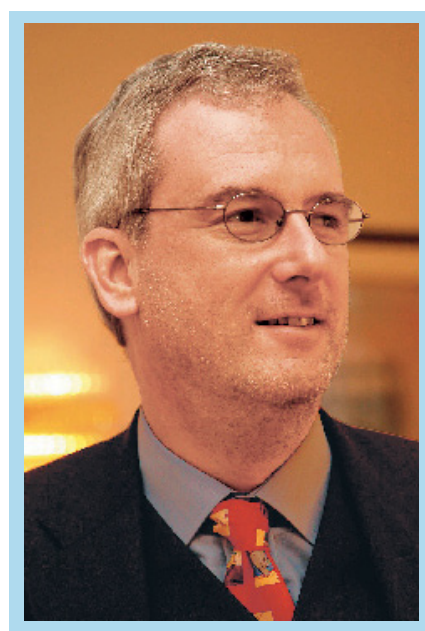

In der Rubrik "Osteoporose Update" referiert und kommentiert Dr. Michael Pfeifer, DVO-Koordinator Osteoporose-Leitlinie Update 2017, für Sie die aktuelle internationale Literatur. Kontakt:

Dr. med. Michael Pfeifer Institut für Klinische Osteologie und Klinik "DER FÜRSTENHOF" Am Hylligen Born 7, 31813 Bad Pyrmont E-Mail: iko_pyrmont@t-online.de ceboarm ist bei diesem Schweregrad einer Osteoporose bedenklich; er wurde mit der geringen Behandlungsrate von Frauen mit Osteoporose im Allgemeinen gerechtfertigt. Ein weiterer Schwachpunkt ist, dass nur der Vergleich mit Placebo verblindet wurde, was mit der fehlenden neutralen Verpackung von Teriparatid erklärt wurde.

Primärer Endpunkt der Studie war der Anteil von neuen vertebralen Frakturen im Verlauf der 18-monatigen Behandlung. Er trat, wie Paul Miller vom Colorado Center for Bone Research in Lakewood, Colorado berichtete, im Placebo-Arm bei 30 Patienten (4,22\%) auf, unter der Behandlung mit Teriparatid dagegen nur bei 6 Patienten $(0,84 \%)$ und unter der Behandlung mit Abaloparatid nur bei 4 Patienten $(0,58 \%)$ auf. Im Vergleich zu Placebo war die Frakturrate um $86 \%$ vermindert. Die Hazard Ratio von 0,14 war mit einem 95\%-Konfidenzintervall von 0,05 bis 0,39 hoch signifikant. Im Vergleich zu Teriparatid dürfte es keinen signifikanten Unterschied gegeben haben.

Bei den nicht-vertebralen Frakturen war der Vorteil von Abaloparatid nicht so eindeutig. Im Placebo-Arm kam es zu 33 Frakturen $(4,7 \%)$, unter der Behandlung mit Teriparatid waren es 24 Frakturen (3,3\%) und unter der Behandlung mit Abaloparatid 18 Frakturen $(2,7 \%)$. Miller und Mitarbeiter errechnen einen Hazard Ratio von 0,57, die mit einem $95 \%$ Konfidenzintervall von 0,32 bis 1,00 nicht signifikant war. Möglicherweise wären die Ergebnisse bei einer größeren Teilnehmerzahl eindeutig ausgefallen.

Beide Wirkstoffe erhöhten die Knochenmineraldichte an Gesamthüfte, Oberschenkelhals und an den Lendenwirbeln, während die Werte im Placebo-Arm sich erwartungsgemäß kaum veränderten und am Oberschenkelhals sogar zurückgingen. Abaloparatid erzielte an allen drei Messorten über den Verlauf der Therapie stets bessere Ergebnisse, sodass auf Dauer und bei einer größeren Fallzahl auch eine niedrigere Knochenbruchrate zu erwarten wäre.

Tatsächlich war die Zahl der schweren osteoporotischen Frakturen (1,5\% versus 3,1\%) deutlich niedriger. Die Hazard-Ratio $(0,45$; $0,21-0,95)$ in diesem sekundären Endpunkt war jedoch nicht signifikant. Ob sich dies bei einer längeren Behandlungszeit geändert hätte muss offen bleiben. Die Behandlungszeit von Teriparatid ist auf 24 Monate begrenzt. Bei Ratten war es unter der Langzeittherapie 
zu einer erhöhten Inzidenz von Osteosarkomen gekommen. Da dies eine mögliche Folge der anabolen Wirkung sein könnte, dürften die Zulassungsbehörden die Anwendungszeit von Abaloparatid ebenfalls begrenzen.

Aufgrund der Unterschiede im Wirkungsmechanismus kam es unter der Behandlung mit Abaloparatid seltener zur Hyperkalzämie (3,6\% versus $6,4 \%)$, was ein Vorteil hinsichtlich der Verträglichkeit sein könnte. Andererseits kam es im Abaloparatidarm häufiger zu
Behandlungsabbrüchen (9,9\% versus 6,8\%). Die häufigsten Ursachen waren Nausea $(1,6 \%)$, Schwindelgefühle, Kopfschmerzen $(1,0 \%)$ und Palpitationen (0,9\%). Schwere Komplikationen, die einer Zulassung entgegen stehen, traten nicht auf.

Der Hersteller hat sowohl bei der amerikanischen Arzneimittelbehörde FDA als auch bei der europäischen Arzneimittel-Agentur (EMA) eine Zulassung beantragt, die er aller Voraussicht nach auch erhalten wird. Die spannende- re Frage ist, zu welchem Preis das Mittel eingeführt wird und ob Konkurrenz in diesem Bereich eventuell die Therapie erschwinglicher machen könnte.

Quelle: Miller PD et al. Effect of Abaloparatide vs Placebo on New Verterbal Fractures in Postmenopausal Women With Osteoporosis: A Randomized Clinical Trial. JAMA 2016; 316(7): 722-733.

Evidenzgrad nach SIGN: 1++

Die Gesamtzahl der klinischen Frakturen sank von 13,0\% (266 von 2047 Patienten) auf 9,7\% (198 von 2046 Patienten). Dies entspricht einem relativen Rückgang um $27 \%$ (Hazard Ratio 0,73; 0,61-0,88).

Diese günstigen Ergebnisse wurden jedoch überschattet von einem Anstieg kardiovaskulärer Ereignisse: Im ersten Jahr erlitten unter der Behandlung mit Romosozumab 50 von 2040 Patienten (2,5\%) ein schweres kardiovaskuläres Ereignis (Herzinfarkt, Schlaganfall). Unter der Behandlung mit Alendronsäure waren nur 38 von 2014 Patienten (1,9\%) betroffen.

Es ist derzeit unklar, was die kardiovaskulären Ereignisse verursacht hat. Die Erklärungen reichen von einer Schutzfunktion von Sklerostin in den Blutgefäßen bis zu einer gefäßprotektiven Wirkungen von Alendronsäure, die zu einem scheinbaren Anstieg kardiovaskulärer Ereignisse geführt haben könnte. Das Fehlen des Sicherheitssignals in der FRAME-Studie spricht für eine zweite Erklärung. Die FDA will vor einer endgültigen Entscheidung die Ergebnisse der BRIGE-Studie abwarten, die derzeit die Wirkung von Romosozumab bei Männern mit Osteoporose untersucht. chen und zum anderen die kumulative Inzidenz von klinischen Frakturen - non-vertebrale und symptomatische vertebrale Wirbelfrakturen.

In beiden Endpunkten überzeugte Romosozumab: In der „Romosozumab-gefolgt-vonAlendronsäure"-Gruppe kam es bei 127 von 2046 Patientinnen zu Wirbelfrakturen (6,2\%). Dagegen traten in der "Alendronsäure-gefolgt-von-Alendronsäure"-Gruppe bei 2047 Patienten 243 Wirbelbrüche auf (11,9\%). Kenneth Saag von der Universität von Alabama in Birmingham ermittelte einen relativen Rückgang um $48 \%$. Die „Risk Ratio" betrug 0,52 und war mit einem $95 \%$-Konfidenzintervall von 0,40 bis 0,66 hoch signifikant.
Quelle 1: Cosman F et al. Romosozumab Treatment in Postmenopausalen Women with Osteoporosis (FRAME-Study). N Engl J Med 2016; 375: 1532-43. DOI: 10.1056/NEJMoa160948

\section{Evidenzgrad nach SIGN: 1++}

Quelle 2: Saag KG et al. Romosozumab or Alendronate for Fracture Prevention in Women with Osteoporosis (ARCH-Study). N Engl J Med 2017; 377: 1417-27. DOI: 10.1056/NEJMoa1708322

Evidenzgrad nach SIGN: 1++ 